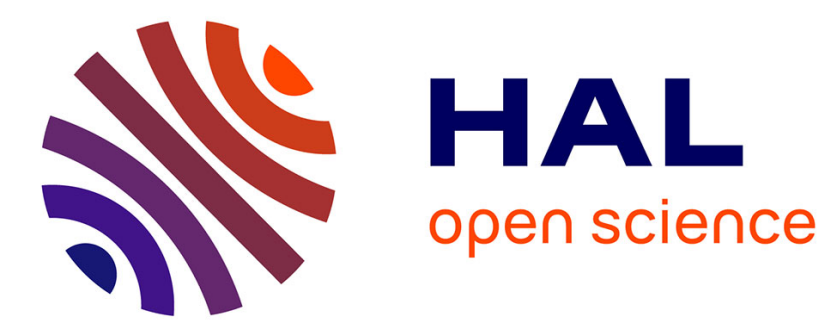

\title{
Les enjeux des paysages d'eau dans la construction du Pays des Mille Étangs
}

Denis Mathis

\section{To cite this version:}

Denis Mathis. Les enjeux des paysages d'eau dans la construction du Pays des Mille Étangs. Revue Géographique de l'Est, 2016, 56 (1-2), 10.4000/rge.5663 . hal-02813689

\section{HAL Id: hal-02813689 \\ https://hal.science/hal-02813689}

Submitted on 6 Jun 2020

HAL is a multi-disciplinary open access archive for the deposit and dissemination of scientific research documents, whether they are published or not. The documents may come from teaching and research institutions in France or abroad, or from public or private research centers.
L'archive ouverte pluridisciplinaire HAL, est destinée au dépôt et à la diffusion de documents scientifiques de niveau recherche, publiés ou non, émanant des établissements d'enseignement et de recherche français ou étrangers, des laboratoires publics ou privés. 


\section{Les enjeux des paysages d'eau dans la construction du Pays des Mille Étangs}

The stakes of the waterscapes in the construction of the Pays des Mille Etangs Herausforderungen der Teichlandschaften am Beispiel des Gebiets des Mille Etangs

Denis Mathis

\section{OpenEdition}

\section{Journals}

Electronic version

URL: http://journals.openedition.org/rge/5663

ISSN: 2108-6478

\section{Publisher}

Association des géographes de l'Est

Printed version

Date of publication: 22 April 2016

ISSN: 0035-3213

\section{Electronic reference}

Denis Mathis, «Les enjeux des paysages d'eau dans la construction du Pays des Mille Étangs », Revue Géographique de l'Est [Online], vol.56 / n 1-2 | 2016, Online since 22 April 2016, connection on 30 April 2019. URL : http://journals.openedition.org/rge/5663

This text was automatically generated on 30 April 2019.

Tous droits réservés 


\section{Les enjeux des paysages d'eau dans la construction du Pays des Mille Étangs}

The stakes of the waterscapes in the construction of the Pays des Mille Etangs Herausforderungen der Teichlandschaften am Beispiel des Gebiets des Mille Etangs

Denis Mathis

1 Les "pays d'étangs" sont des territoires ruraux construits sur la perception d'une identité paysagère forte : celle de la maitrise de l'eau au cœur d'un territoire aménagé souvent de longue date par l'homme. C'est un territoire d'eau, au sens défini par S. Ghiotti (2007), et organisé à partir de petites ou de grandes retenues d'eau. Les étangs ont été façonnés par la mise en œuvre d'un système piscicole le plus souvent complémentaire, mais aussi parfois antagoniste, au système agraire ou sylvicole.

Pour le plateau des Mille Étangs, dans les Vosges Saônoises, il s'agit très nettement d'une complémentarité de mise en valeur. Les "Mille Étangs " ${ }^{1}$ forment un petit territoire d'étangs couvrant $220 \mathrm{~km}^{2}$, perché sur un plateau granitique aux altitudes moyennes supérieures à 600 mètres. Ce versant Sud-Ouest du massif vosgien est marqué par une forte empreinte glaciaire provoquée par les déversements du glacier de la Moselle au Quaternaire, au-delà de la ligne de crêtes (Flageollet, 2002). Le paysage post-glaciaire de ce plateau évoque les milieux nordiques, d'où son surnom de "Petite Finlande " par analogie avec les « Mille Lacs » finlandais².

Les zones humides d'altitude ont occupé les bassins de surcreusement et les vallons. Verrous et accumulations de matériel morainique gênent les écoulements et favorisent le développement de marécages et de tourbières. Cet héritage du creusement glaciaire constituait un substrat favorable à la création d'étangs. Ainsi, c'est ce contexte de modelés glaciaires qui a permis de façonner le paysage du plateau en "pays d'étangs ». Les recensements actuels donnent environ 850 étangs et les modes d'exploitation agrosylvo-piscicoles qui ont sécrété ce paysage sont aujourd'hui largement déclinants. Bien 
que son importance soit secondaire dans l'activité économique, l'étang piscicole, marque des terroirs du plateau, est devenu l'élément identitaire et symbolique de ce «pays ». D. Guillaud affirme que "l'étude des différents vestiges archéologiques (...) révèle le regard sur le passé des habitants actuels, lequel souligne les géosymboles, « lieux forts » ou monuments, naturels ou construits, structurant leur territoire » (2008). Pour le plateau des Vosges Saônoises, les étangs sont les vestiges des anciens systèmes agraires. Ils, structurent et maillent l'espace, façonnant l'identité actuelle du plateau. En quelque sorte l'étang est devenu ce géosymbole : «c'est-à-dire un lieu, un itinéraire, un espace, qui prend aux yeux des peuples et des groupes ethniques, une dimension symbolique et culturelle, où s'enracinent leurs valeurs et se conforte leur identité » (Bonnemaison, 1992).

4 D'un espace rural ordinaire de moyenne montagne, transposable dans d'autres régions de France, on assiste à un glissement d'un territoire rural productif à un espace naturel. Il peut être qualifié "d'authentique » tel que le définit (Delignières, 1998) parce que ce paysage est à la fois marginal et cet espace enclavé donne l'impression d'une région atypique et d'un sentiment de «bout du Monde». Bien que ne représentant que $7 \%$ du territoire, les étangs sont des marqueurs paysagers, générateurs d'identités et par la même de nouvelles fonctionnalités. Ainsi, ils entrent désormais au sein d'un assolement récréatif et de loisirs dans le cadre des mutations de l'espace agricole pour aboutir à une mise en valeur touristique intégrée au Parc Naturel Régional des Ballons des Vosges (PNRBV). Le Plateau des Mille Étangs illustre la «co-évolution entre des systèmes naturels et sociaux $»^{3}$. Inscrite dans le temps long, l'expression visible de cette évolution est le paysage. Ce dernier porte, tel un palimpseste, l'ensemble des vestiges des anciens systèmes, empilant et superposant les formes les plus résilientes dans les nouveaux paysages et effaçant ou délaissant les éléments déclassés, inutiles indices plus ou moins ténus des systèmes antérieurs.

5 L'anthroposystème " Mille Étangs ", par sa production paysagère reflète : pour le plateau, les multiples dynamiques agricoles, architecturées ou encore naturelles, et pour les vallées du Breuchin et de l'Ognon, les déploiements pré-industriels, industriels et postindustriels. Depuis le développement des proto-industries au $\mathrm{XV}^{\mathrm{e}}-\mathrm{XVI}^{\mathrm{e}}$ siècles, la dynamique de construction systémique est orientée vers la gestion cohérente de la ressource en eau. Au moyen d'une démarche rétrospective, nous chercherons à montrer l'importance de la mise en exploitation et de la gestion de la ressource en eau à partir des vestiges hydrauliques les plus emblématiques de ce territoire, à savoir les étangs. Ces constats nous permettront de cerner quelques-uns des enjeux de la préservation de ces hydrosystèmes et de leur prise en compte au sein de projets de développement, de protection ou de renaturation.

\section{Un ancien territoire agricole de moyenne montagne : le Plateau des « Mille Étangs »}

\section{A. Un territoire marqué par l'empreinte glaciaire et l'hydromorphie}

Le Pays des Mille Étangs, fragment des Vosges Saônoises, est un des territoires d'étangs parmi les moins connus et pourtant c'est un des plus originaux des pays d'étangs de l'espace national. Ce haut plateau prolonge une section du versant sud-ouest de la montagne vosgienne. Il constitue un espace atypique puisqu'il s'agit d'un territoire d'étangs ${ }^{2}$, établi en grande partie à des altitudes supérieures à $600 \mathrm{~m}$ et atteignant $720 \mathrm{~m}$ 
à $730 \mathrm{~m}$ au nord-est, au contact de la ligne de crête dominant la vallée de la Moselle (740 m à $750 \mathrm{~m})$. Pour l'essentiel, ce pays d'étangs se situe entre les vallées du Breuchin à l'ouest et de l'Ognon à l'Est.

7 L'hydrosystème organisé autour de ces deux rivières a été façonné par un important aménagement lentique, que ce soit au niveau du plateau ou des hautes vallées des deux bassins hydrographiques. L'abondance d'eau et l'hydromorphie ambiante sont liées à un relief marqué par l'action glaciaire quaternaire. Le Plateau des Mille Étangs a connu deux, voire trois glaciations marquées par le débordement et le déversement du glacier de la haute Moselle (fig. 1).

Figure 1. Croquis des limites des glaciations et de leurs traces dans le paysage

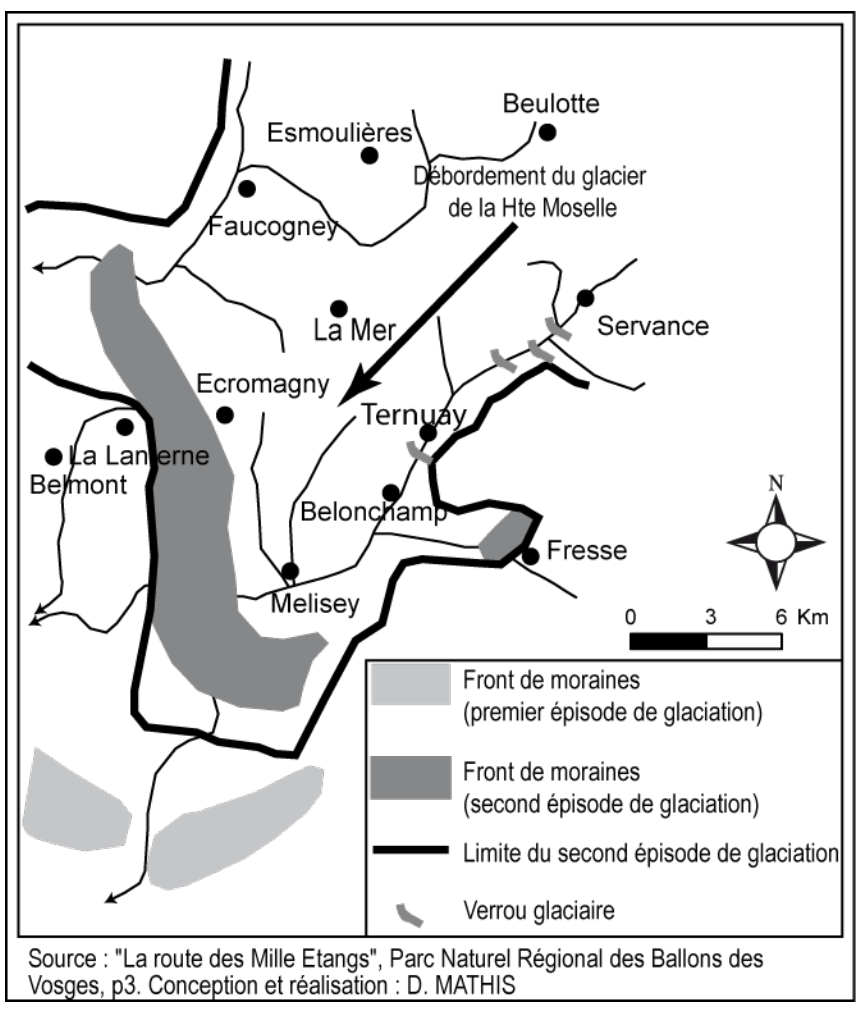

8 Ainsi l'empreinte glaciaire a façonné la topographie de ce plateau, rabotant l'ensemble de la partie sommitale, laissant roches striées, blocs erratiques, roches moutonnées et moraines. Les zones de surcreusement ont été occupées par l'eau produisant une vaste zone humide composée de marais et de tourbières. C'est à partir de ces cuvettes que l'homme a alors aménagé des étangs. La présence de cuvettes de surcreusement en position sommitale des croupes vosgiennes a facilité ces aménagements presque jusqu'à la ligne de crête (730 m d'altitude). Depuis le sommet, les étangs s'organisent en longs chapelets, occupant les cuvettes, depuis la ligne de crête jusqu'aux rebords du plateau, pour s'écouler ensuite vers les vallées de l'Ognon et du Breuchin.

\section{B. Le désordre de la déprise agraire}

9 Ce vaste plateau est aujourd'hui en grande partie forestier. Le taux de boisement est relativement important. La forêt totalise 11000 hectares du territoire de la Communauté de Communes du Pays des Mille Étangs (CC-1000 Étangs), dont 6400 ha de forêts privées 
appartenant à 2000 propriétaires différents. Cette forêt est en phase d'expansion et de reconquête. Elle témoigne, comme dans la plupart des plateaux et périphéries du massif vosgien, du désordre de la déprise des anciens systèmes agraires (Dupré, 2005, Mathis, 2015). Ainsi, la CC-1000 Étangs souligne que ces paysages connaissent «des mutations profondes se traduisant sur le terrain par la fermeture et la banalisation du paysage à cause d'une déprise agricole croissante, la dégradation d'un patrimoine bâti ancien par abandon... $»^{4}$. La Surface Agricole Utile (S.A.U.) couvre encore $25 \%$ du territoire de la CC-1000 Étangs, essentiellement dans la vallée du Breuchin et de l'Ognon. Pour les communes du plateau, le déclin agricole a conduit également à une déprise de la présence humaine. Ce vide est tout autant le résultat des difficultés d'une agriculture de montagne, avec un ancien agrosylvo-stagnosystème ${ }^{5}$ à présent obsolète, que celui de l'enclavement. La logique de fermeture du paysage et de recomposition du faciès forestier aujourd'hui dominant est la marque du déclin. La recomposition de la forêt s'effectue par reboisement, mais aussi par extension de la friche. Cette logique de fermeture du paysage et de déprise anthropique donne l'impression d'un gradient dans «l'habiter », entre la nature "sauvage » et "déserte", les habitats secondaires de loisirs et de tourisme qui constituent un " habiter " temporaire, et enfin les villages, hameaux et fermes, noyaux d'un " habiter " permanent. Cette lecture s'inscrit au sein d'un plateau qui est un « désert humain ». Ainsi, une commune emblématique telle que Beulotte-Saint-Laurent (1 420 hectares) n'abrite guère plus de 70 habitants répartis dans le village et ses écarts. Les villages, les écarts peuvent être qualifiés de lieu d'érème, faisant ainsi référence à A. Berque (2011) dans son analyse des relations entre « le rural, le sauvage, l'urbain ».

\section{L'étang : un terroir à forte valeur symbolique et culturelle}

10 Au sein du PNRBV, le Plateau des Mille Étangs apparaît comme une entité territoriale marginale. En effet, par rapport aux autres composantes territoriales du parc, et notamment aux espaces des crêtes et des ballons vosgiens de Lorraine et d'Alsace ou pour le versant franc-comtois avec le Ballon de Servance, l'attractivité touristique du plateau, isolé et enclavé, apparaît bien secondaire. Les étangs sont des éléments constitutifs s'inscrivant dans une lecture d'un " paysage ordinaire ", voire dans le contexte de déprise qui peut être intégré au "tiers paysage » défini par G. Clément (2004). Pourtant, l'enclavement, le vide, l'érème constituent une chance de développement touristique. Les anciennes fermes, isolées au sein de clairières, de prairies avec leurs petits étangs, constituent, toutes proportions gardées, un paysage proche des forêts ponctuées de lacs des espaces baltiques ou canadiens (fig. 2). Elle renvoie une certaine image d'authenticité, voire d'exotisme, à quelques heures des espaces urbains. Elle traduit le désir, pour cette société urbaine de ses « envies de campagne » (Husson J-P., 2008), d'individualisme et de recherche de nature, loin des campagnes citadinisées. 


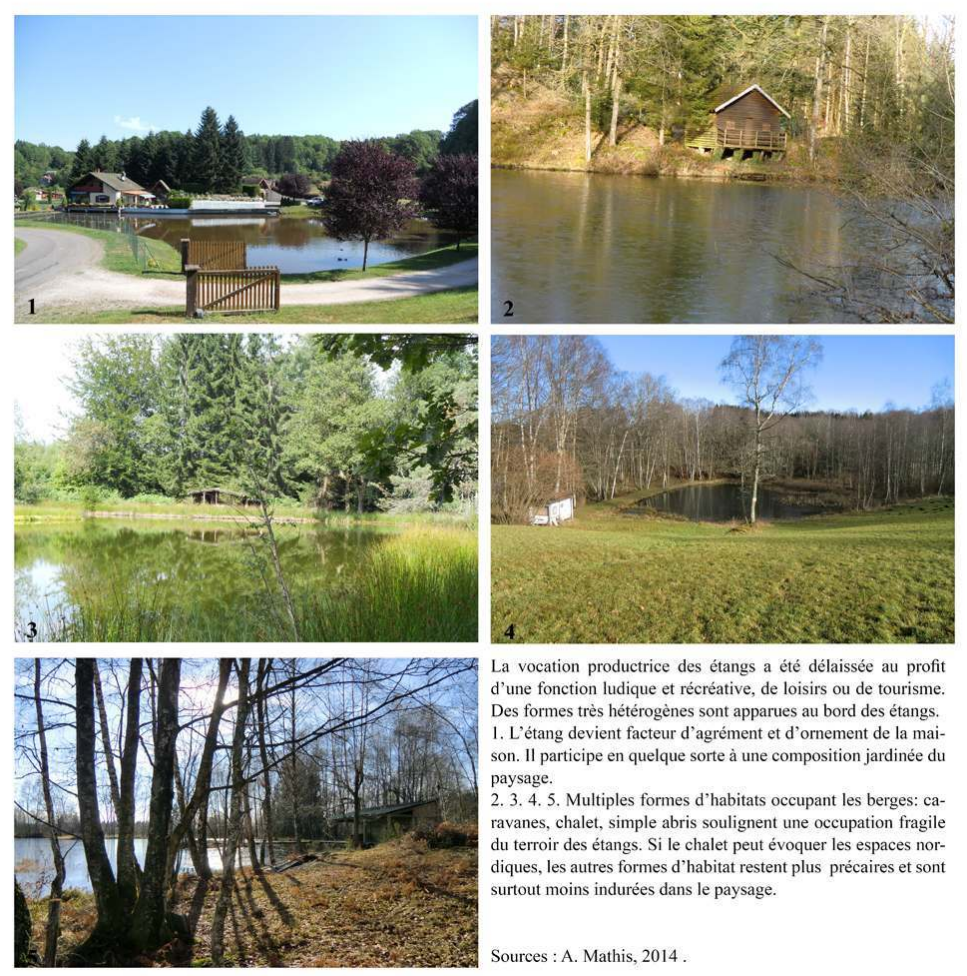

11 L'enclavement et le «désert " sont un facteur favorable au développement local. Le regard porté sur le Plateau des Mille Étangs est désormais culturel et social et de moins en moins lié à une activité économique. Ce glissement dans la perception des paysages du plateau avec ses étangs illustre, le processus évolutif décrit par Y. Luginbühl, suivant lequel «le paysage d'abord assimilé à la campagne s'est rapproché, dans les représentations sociales, de la nature » (2012, p.187). Ainsi pour le public, comme il existait une fusion entre l'agriculture et le paysage, il existe désormais une fusion entre la nature et le paysage.

Dans le cas des Mille Étangs, comme souvent pour les territoires d'étangs marqués par la déprise agraire, ce développement des lieux de loisirs et des résidences secondaires se trouve confortée par la faiblesse des prix du foncier et de l'immobilier. Cette dernière a permis l'installation dans des résidences secondaires de nouveaux habitants originaires de Suisse alémanique ou du land de Bade-Wurtemberg. Déprise et friche ont fait éclore un paysage à forte valeur de naturalité. La faible valeur foncière et immobilière a permis de faire naître un espace touristique et récréatif basé sur un parc locatif et de résidences secondaires ${ }^{6}$ et sur la représentation et la sémiologie des étangs plus que sur leur usage. Ainsi, dans un contexte où " le paysage rural a été pendant des siècles le résultat non recherché de l'activité agricole et considéré comme un sous-produit » (Brossier et al.,2008), le terroir des étangs se retrouve sensiblement réévalué.

Le développement touristique est une opportunité pour les espaces ruraux car il crée un nouvel " assolement récréatif », comme le souligne Y. Le Caro (2012). Pour les dernières fermes du plateau, il s'agit d'exploiter la valeur paysagère des étangs dans l'émergence d'une activité complémentaire. En cela, les étangs sont désormais une ressource pour le développement ou simplement le maintien de cet assolement récréatif. Aussi la question de la permanence des étangs est aujourd'hui fondamentale pour la construction du projet 
territorial du plateau. Elément identitaire, au point de donner son nom au plateau, ce géosymbole est menacé car l'activité piscicole, et plus particulièrement la carpiculture, est sur le déclin. Sur les 850 étangs seuls 70 connaissent encore un assec régulier. Essentiellement aux mains de particuliers, cette activité économique extensive et peu productive est un héritage d'ancienne pratique alimentaire. Actuellement la filière recherche de nouveaux débouchés, avec le développement de l'élevage des truites ou l'introduction de l'écrevisse à pattes rouges, malgré les enjeux de biodiversité. Le maintien des pêches est un des facteurs essentiels pour assurer la résilience, c'est-à-dire la capacité du stagnosystème à retrouver un équilibre partiel de son fonctionnement stabilisant les paysages d'étangs au sein du désordre de la nature et de la déprise agraire.

\section{Les systèmes « Mille Étangs » et la gestion de l'eau}

14 Les étangs mis en place progressivement, constituent l'élément identitaire du plateau et reflètent l'empilement de plusieurs strates systémiques. Ils marquent et maillent un territoire d'eau. Ils permettent d'assurer la gestion de l'hydromorphie et sont destinés à faire de cet excès d'eau une ressource valorisable.

\section{A. Aux origines d'un pays d'étangs}

Comme souvent la tradition et le récit historique rapportent un aménagement très ancien de ce territoire d'étangs. Les érudits et historiens s'accordent pour une mise en valeur piscicole de ce territoire à partir du $\mathrm{XI}^{\mathrm{e}}$ siècle en liaison avec l'installation d'établissements religieux et particulièrement les abbayes de Lure et de Luxeuil-les-Bains et secondés par les seigneurs de Faucogney.

L'essor de la pisciculture sur le plateau est présenté comme le principal moteur de ces aménagements d'étangs. En fait, si on considère le $\mathrm{XI}^{\mathrm{e}}$ siècle comme fondement chronologique, le développement de la pisciculture est une conséquence de la conquête agricole du plateau. En effet, la faible productivité de cette pisciculture de montagne ne peut faire de cette celle-ci qu'une activité complémentaire. Il faut voir dans l'essor de la pisciculture la conséquence du développement des étangs et non l'origine de leur création.

17 En fait, les travaux d'aménagement des étangs sont liés à la mise en valeur des terres agricoles et notamment à la bonification des prairies. La mise en valeur du plateau sur le modèle agro-piscicole est liée à l'augmentation de la pression anthropique, correspondant peut-être au passage d'un élevage transhumant et d'une occupation temporaire à un élevage et une occupation permanente.

La nécessité de ressources plus abondantes au sein de ce territoire contraint à bonifier les terres en luttant contre l'hydromorphie. Il a été nécessaire de réorganiser les «zones humides ", de favoriser les écoulements afin d'augmenter les pâturages et les prairies de fauche. Le modèle agricole qui s'est développé repose alors sur les ressources variées de l'élevage bovin laitier, de l'activité forestière (bois, charbon de bois et écorces) et de l'activité piscicole.

19 Toutes ces productions sont destinées à la plaine et leurs ventes permettaient d'acquérir les ressources alimentaires non produites sur le plateau. Bien qu'isolés sur le plateau, les fermes et hameaux ne peuvent vivre en autarcie et sont liés aux échanges avec la plaine. 
À ce titre, les deux vallées du Breuchin et de l'Ognon constituent les deux axes majeurs d'échanges. Le système piscicole a été probablement construit en plusieurs étapes.

En effet, si l'origine des étangs remonte au XI ${ }^{\mathrm{e}}$ siècle, en revanche, la diffusion et l'essor de la carpiculture sont plus tardifs et ne remontent au plus tôt qu'au XIII ${ }^{e}$ siècle (Benarrous et Marinval, 2006). Aussi, s'il est possible de relever une activité piscicole antérieure à la diffusion de la carpe, cette dernière ne repose que sur la pisciculture de la truite. Ce modèle est propre aux pays d'étangs des marges vosgiennes. En effet, les espaces piscicoles du Pays de Bitche (Lorraine) dans un contexte quelque peu différent ont produit un système productif comparable. L'introduction de la carpe dans les étangs s'inscrirait tel un « seuil technique » dans l'utilisation des ressources du plateau. En effet l'accroissement des ressources alimentaires et commerciales engendré par cette introduction a pu favoriser non seulement l'essor de cette activité complémentaire, mais également être le moteur de la mise en eau de nouveaux étangs.

21 Dans ce contexte d'une agriculture de montagne sur ces "hauts plateaux" aux sols particulièrement médiocres et où la vie rurale était difficile, cette pisciculture constituait un complément pour les exploitations agricoles. Désormais, cette activité devient indispensable et utilise les étangs réservoirs, accélérant leur mise en place au point de conquérir les zones humides. Ce mécanisme de conquête du plateau et de mobilisation des ressources en eau pour la pisciculture ne doit pas faire oublier que dans ces étangs oligotrophes, les eaux acides et pauvres en éléments nutritifs ont de faibles rendements de l'ordre de $60 \mathrm{~kg} / \mathrm{ha} / \mathrm{an}$ contre $150 \mathrm{~kg} / \mathrm{ha} /$ an dans la pisciculture assolée de plaine en Lorraine.

L'observation des cartes anciennes permet de constater une dynamique d'extension des étangs entre le XVIII ${ }^{e}$ et le XIX ${ }^{e}$ siècle. Il est probable que l'affirmation de l'agro-sylvostagnosystème du plateau ne corresponde en fait qu'à cette période du plein-rural.

\section{B.- Un agro-sylvo-stagnosystème}

Loin d'avoir été une contrainte, l'excès d'eau sur le plateau semble avoir constitué une opportunité pour assurer le développement agricole. Seule faisait défaut la maitrise des techniques hydrauliques. Probablement développées et diffusées par les moines, elles ont été à l'origine de la mise en valeur agricole. Ainsi comme souvent pour les pays d'étangs, le rôle des communautés monastiques est présenté comme capital. Toutefois, on peut se demander si la faiblesse des sources anciennes, relatives à l'aménagement stagnustre, n'entraîne pas une surévaluation historique générale de ce rôle, surtout si on considère le nombre et la petite taille des étangs ${ }^{7}$ aménagés sur ce plateau. Ne faudrait-il pas plutôt apprécier le rôle d'une communauté agricole déterminée à accroître ses ressources et s'inscrivant dans le cadre d'un aménagement progressif pluri-générationnel qui a conquis les zones humides (tourbières et vallons) ? Finalement ce phénomène serait alors comparable à la conquête des versants des vallées vosgiennes, sauf qu'au lieu d'être un processus s'inscrivant de bas en haut, il s'agit peut-être dans le cas du plateau d'un aménagement qui débute à partir des hameaux et qui se déploie ensuite vers l'amont et l'aval des cours d'eau.

Ce processus serait lié à la mise en place de l'habitat dispersé des fermes et de conquête agraire. Cette expansion, comparable à un front pionnier, installe de nouvelles exploitations et s'accompagne alors de l'aménagement d'étangs. Ces derniers forment des réserves d'eau disponibles pour l'agriculture, notamment pour le développement de 
l'irrigation. En effet, la maîtrise de l'eau était nécessaire au développement de prairies irriguées afin d'accélérer le démarrage de la saison végétative et la pousse de l'herbe en hâtant le "réchauffement" des sols après les gelées de mars à mai. Cette technique pouvait être également utilisée avant les regains pour faire face à des périodes sèches, voire après les regains (octobre-novembre), pour prolonger la saison végétative. L'augmentation des ressources fourragères par l'irrigation permet la nourriture du cheptel pendant la saison hivernale. Cette technique a été largement diffusée dans les zones de moyennes montagnes et le massif vosgien en particulier (Cabouret, 1999), ou dans la montagne limousine (Ardillier-Carras, 2009). Pour le Plateau des Mille Étangs, c'est cette situation sommitale de l'irrigation qui façonne l'organisation paysagère et systémique du territoire. Ainsi, les prés irrigués se trouvent en aval de l'étang ou des étangs qui bordent le plus souvent les bâtiments de ferme. L'abondance de l'eau sur le plateau a permis de construire des terroirs irrigués et des clairières culturales autour des étangs. En aval de la retenue partent des rigoles de distribution et d'épandage qui se déversent dans les prairies. L'utilisation des retenues d'eau pour la pisciculture a été une activité opportuniste. Elle s'accompagne de l'aménagement de "carpières", ces petits bassins dans lesquels les poissons étaient entreposés après la pêche afin de les faire dégorger. Elle a créé une ressource complémentaire.

Les étangs du plateau renforçaient également les réserves d'eau disponibles pour les vallées de l'Ognon et du Breuchin, où les agriculteurs irriguaient également les prairies pour les mêmes raisons que sur le plateau. Ainsi, l'aménagement des étangs a créé des solidarités entre le plateau et les vallées. Ces dernières ont été renforcées au fil du temps par l'essor de proto-industries, puis de l'industrie dans la région. Elle s'est appuyée sur l'utilisation de la force hydraulique à partir du véritable château d'eau que représente le plateau. Les étangs sont donc également des étangs-réservoirs à vocation agricole et industrielle.

Il faut considérer que si cette logique d'aménagement d'étangs relève d'un individualisme agricole, en revanche la gestion de la ressource en eau, compte tenu de l'organisation en chapelet des étangs, induit une démarche collective (fig. 3). Il est probable que cette dernière ait été facilitée par l'étagement des étangs des crêtes vosgiennes jusqu'aux vallées encaissées du Breuchin et de l'Ognon. En effet, le mécanisme d'irrigation agricole, lié au démarrage végétatif, induit pour les vallées puis le plateau une vidange de l'aval vers l'amont des cours d'eau. Ainsi, au fur et à mesure des vidanges, les étangs situés à l'aval récupèrent les eaux lâchées en amont, ce qui permet d'assurer dans les vallées une irrigation régulière au printemps puis à l'automne. Pour les fermes situées en amont, la fonte des neiges au printemps et au début de l'été permet de compenser le renouvellement des eaux. Ainsi, la pratique agricole permet d'assurer un renouvellement et une gestion cohérente des eaux des étangs sommitaux jusqu'aux fonds de vallées de l'ognon et du Breuchin. 
Figure 3. Un paysage de fermes isolées avec son terroir d'étangs.

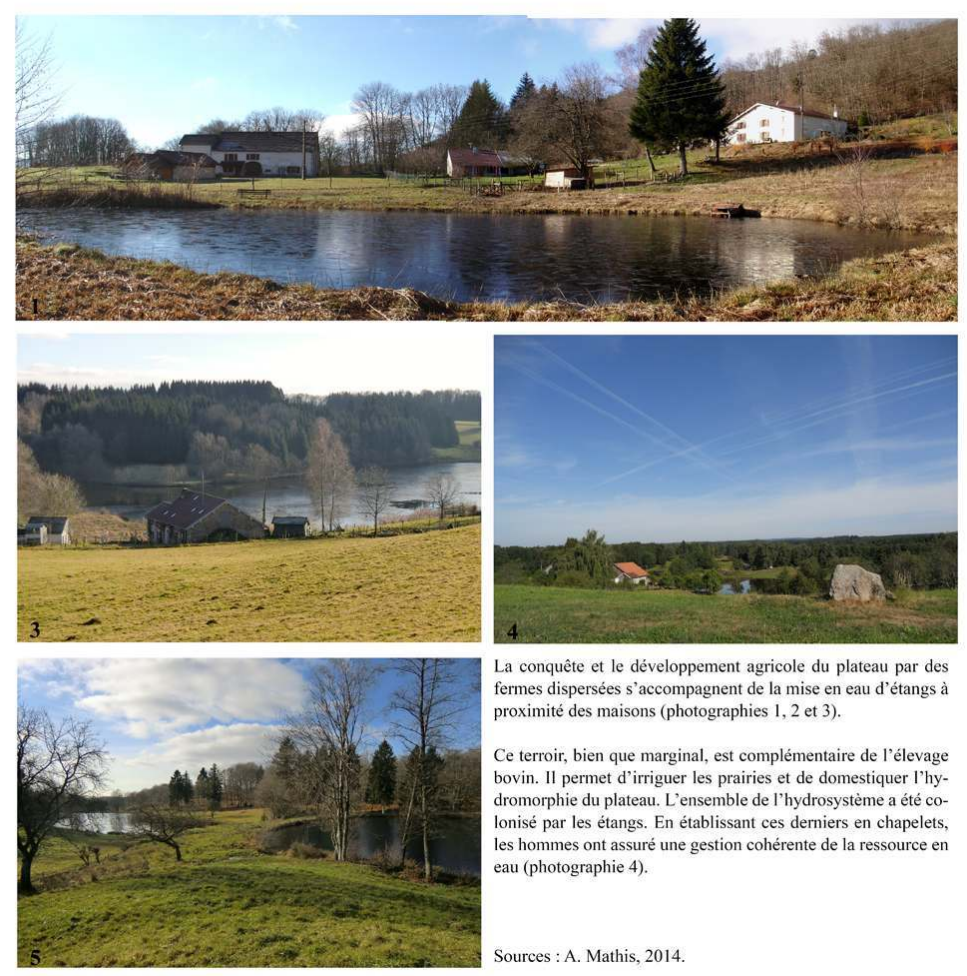

\section{L'utilisation de la ressource en eau par l'industrie}

La régulation des hydrosystèmes de l'amont vers l'aval a permis d'assurer une régularité du débit des cours d'eau. Nous avons suggéré que la construction des étangs s'était accrue aux XVIII ${ }^{\text {-XIX }}{ }^{\text {e }}$ siècles. L'essor agricole accompagnait alors un essor industriel. La gestion ancienne de l'hydrosystème et de la ressource en eau était favorable au développement du secteur pré-industriel, qu'il s'agisse de moulins ${ }^{8}$, de scieries ${ }^{9}$ ou de foulons. Ainsi A. Perrier signalait que «en dehors des papeteries on ne trouvait le long des rivières que quelques moulins foulon pour la préparation de la laine et des teintureries" et que "l'utilisation industrielle des rivières ne remonte guère qu'au siècle dernier exception faite pour les papeteries de la vallée du Breuchin dont on retrouve la trace jusqu'au XV siècle » (1925). L'industrie verrière a profité également de la mise en exploitation et des défrichements du plateau; elle a pu profiter de l'exploitation de la tourbe lors de la mise en eau des étangs.

Progressivement la ressource en eau mobilisée et mobilisable à partir des étangs va servir à l'essor industriel du XIX e siècle. Ainsi, note A. Perrier (1925), c'est « vers 1850-1860 (que) des industriels d'origine vosgienne ou alsacienne créent des tissages mécaniques ou même des filatures à Haut-du-Them sur de petits affluents de Ognon et la Longine sur un petit affluent du Breuchin (...), les propriétaires des anciennes papeteries remplacent la fabrication du papier par la filature et le tissage du coton». Enfin "Quelques années avant la guerre de 1914 des industriels vosgiens ont créé de nouveaux tissages à Servance, à Champagney, à Faucogney (...). Le travail du coton a même fini par absorber les autres industries textiles. Le tissage du droguet (...) qui s'était maintenu longtemps à Melisey a complétement disparu ». Ce second temps de l'essor industriel exploite la ressource en eau disponible dans les vallées et vallons, créant une solidarité entre le plateau qui dispose d'une population abondante et de la ressource en eau et les 
vallées qui constituent les axes de circulation et disposent de l'espace nécessaire à l'installation d'établissements industriels. La métallurgie et surtout le textile se sont développés, se superposant, voire effaçant la strate pré-industrielle. Ainsi, à Servance la commune a compté13 moulins, 5 scieries et 2 tissages. À Corravillers, c'est la forge Clément ${ }^{10}$ spécialisée dans la production de matériel agricole et notamment de charrue.

La complémentarité de gestion agricole et industrielle de l'eau a permis de compléter les hydrosystèmes assurant le fonctionnement territorial. Aujourd'hui, le déclin industriel des vallées s'accompagne, comme dans le cas de la forge Clément, d'une démarche patrimoniale qui prolonge finalement celles mises en œuvre pour les anciens moulins. Elle permet de valoriser cet héritage dans le cadre d'un projet territorial dont le fil directeur reste l'eau. C'est l'origine de la construction du réseau thématique de la « force hydraulique du PNRBV. Finalement, le Plateau des Mille Étangs constitue un territoire d'eau dont les hydrosystèmes portent les héritages multiples des systèmes agricoles et industriels. Aujourd'hui les uns et les autres sont sur le déclin et rendent disponible la ressource en eau pour d'autres usages. C'est la permanence même de la gestion de l'eau qui est finalement à réévaluer (fig.4).

Figure 4. L'évolution paysagère des Mille Étangs

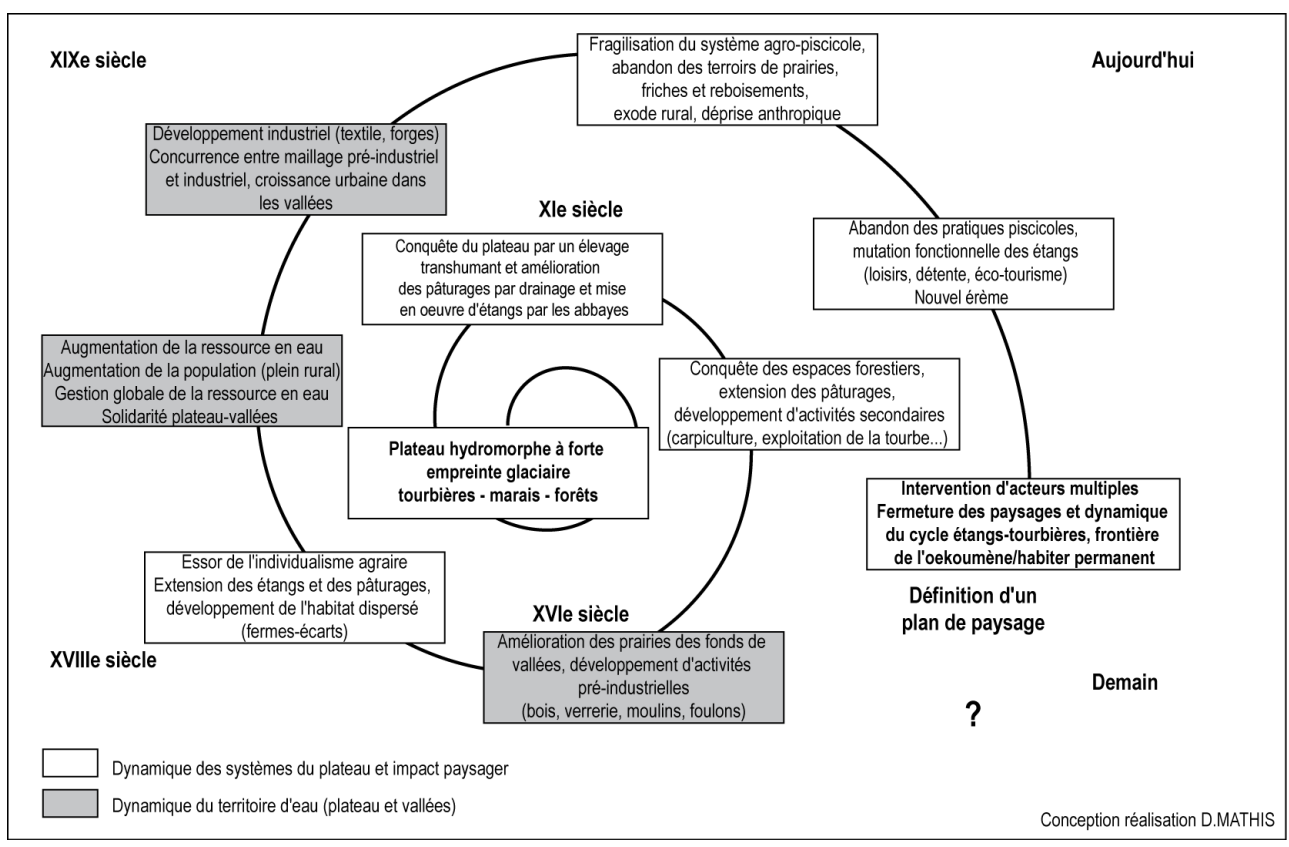

\section{III.- Médiation autour des paysages d'eau}

Le plateau des Mille Étangs a connu, comme de nombreux territoires façonnés autour de la gestion de l'eau, une transformation progressive de son image et de ses représentations. La déprise anthropique, qu'elle soit quantitative ou productive, a entrainé une transformation du territoire déclinant. La valeur patrimoniale naissante a été favorable à la construction « d'un assolement récréatif » établi sur la recherche de la consommation d'un espace nature. L'ambivalence de la perception des étangs, à la fois nature et culture, est alors un atout dans la définition d'un projet territorial soutenu par le PNRBV. 
31 La question reste aujourd'hui de trouver un équilibre entre la gestion de la part naturelle des étangs et des hydrosystèmes et l'utilisation de la ressource paysagère des étangs dans une politique de développement des espaces touristiques. Cet équilibre reste difficile à mettre en œuvre comme l'ont souligné E. Chiffre et D. Mathis (2015) dans le cas des hydrosystèmes stagnustres au sein du Parc Naturel Régional de Lorraine et du Parc Naturel Régional du Morvan. Pour le cas des Mille Étangs, l'action de la CC-1000 Étangs ou de la Communauté de Communes de la Haute Vallée de l'Ognon est déterminante.

\section{A. Quelle lecture paysagère à l'aune de la déprise de l'assolement agro-stagnustre et de l'émergence d'un terroir récréatif et touristique?}

32 Le paysage constitue le cœur de l'identité de ce territoire rural marginal des Vosges Saônoises. Ainsi comme le souligne C. Juin-Rialland, (2009), l'étude paysagère est « la partie perceptible de la construction sociale et culturelle d'un territoire. C'est une source de savoirs mais aussi un condensé de représentations ". Pour ce territoire, les étangs sont le fruit d'un aménagement de générations d'hommes qui sont parvenus à construire un hydrosystème complexe, occupant vallées et plateaux. Ces étangs sont les géosymboles du territoire, malgré le déclin de l'industrie et de l'agriculture délaissant ces espaces aménagés.

La dynamique actuelle, marquée par la déprise anthropique, fragilise l'ensemble des terroirs agricoles, avec finalement et en dernier ressort, les terroirs des étangs. En effet, le déclin agricole a favorisé une forte reconquête forestière. Cette dernière constitue un facteur de fragmentation et de nucléarisation de l'espace du plateau. Malgré le nombre considérable d'étangs, ce dernier offre à l'observateur de moins en moins perceptiblement un "paysage d'eau ». Trop petits, isolés au milieu de clairières, ils ont tendance à être enchâssés au sein d'un massif forestier en extension. Ils participent au maintien d'un « espace ouvert » au sein d'un paysage qui se referme. Inscrits au cœur des massifs forestiers, ils constituent des trouées paysagères que l'on peut qualifier de « clairières en eau ".

La reconquête forestière n'est qu'un aspect de la dynamique paysagère en cours. En effet, sur une échelle de temps plus longue, les étangs ont tendance, lorsqu'ils ne sont plus entretenus, à s'inscrire naturellement dans un processus de disparition. Le déclin de l'activité piscicole, l'absence d'entretien des digues entraînent la disparition inéluctable de l'étang qui peut d'ailleurs être immédiate. C'est le cas lorsque la digue est crevée ou la bonde ou le moine abandonnés. Ce déclin peut s'inscrire à plus long terme par la transformation de l'étang en tourbière. Il s'agit d'un processus naturel qui peut être vu comme faisant partie du cycle des étangs. En fait, marais et tourbières ponctuaient le plateau avant les aménagements des étangs. De nombreuses cuvettes d'étangs sont des tourbières aménagées et exploitées. D'ailleurs, le plateau a toujours conservé ses tourbières, dont l'exploitation a été une des activités secondaires des habitants du plateau comme à Beulotte-Saint-Laurent. À ce titre l'hydrosystème du plateau joue le rôle de réservoirs de biodiversité ${ }^{11}$.

Aujourd'hui, l'abandon de l'entretien et des assecs dans ce milieu de moyenne montagne, favorise la prolifération de la végétation. Un radeau de plantes se développe alors occupant progressivement le plan d'eau. La décomposition végétale est ralentie par les eaux pauvres en oxygène et aux températures faibles. Progressivement ce radeau végétal 
se tisse, donnant naissance à une tourbière flottante, puis une tourbière plate, suivie d'une tourbière bombée et pour finir une tourbière boisée (fig.5). Ce processus voit son aboutissement dans la fermeture, à plus ou moins longue échéance, des clairières stagnustres. Cette dynamique entraîne une perte de valeur paysagère au profit d'une augmentation de l'intérêt écologique et de la biodiversité. Ce cycle est amorcé pour de nombreux étangs, et il est encouragé par le PNRBV dans le cadre d'une protection et d'une promotion de la biodiversité autour de ces milieux sensibles et remarquables. Paradoxalement, il s'agit d'un atout et d'une vitrine dans la présentation de "l'espace nature » du plateau. Il ne faut pas oublier, comme le souligne D. Mathis (2014) pour le Pays des Étangs, que c'est «l'aspect intemporel des paysages d'eau et de forêts " qui «crée un paysage nature ".

Figure 5. Les paysages d'étangs au sein du cycle de la déprise anthropique
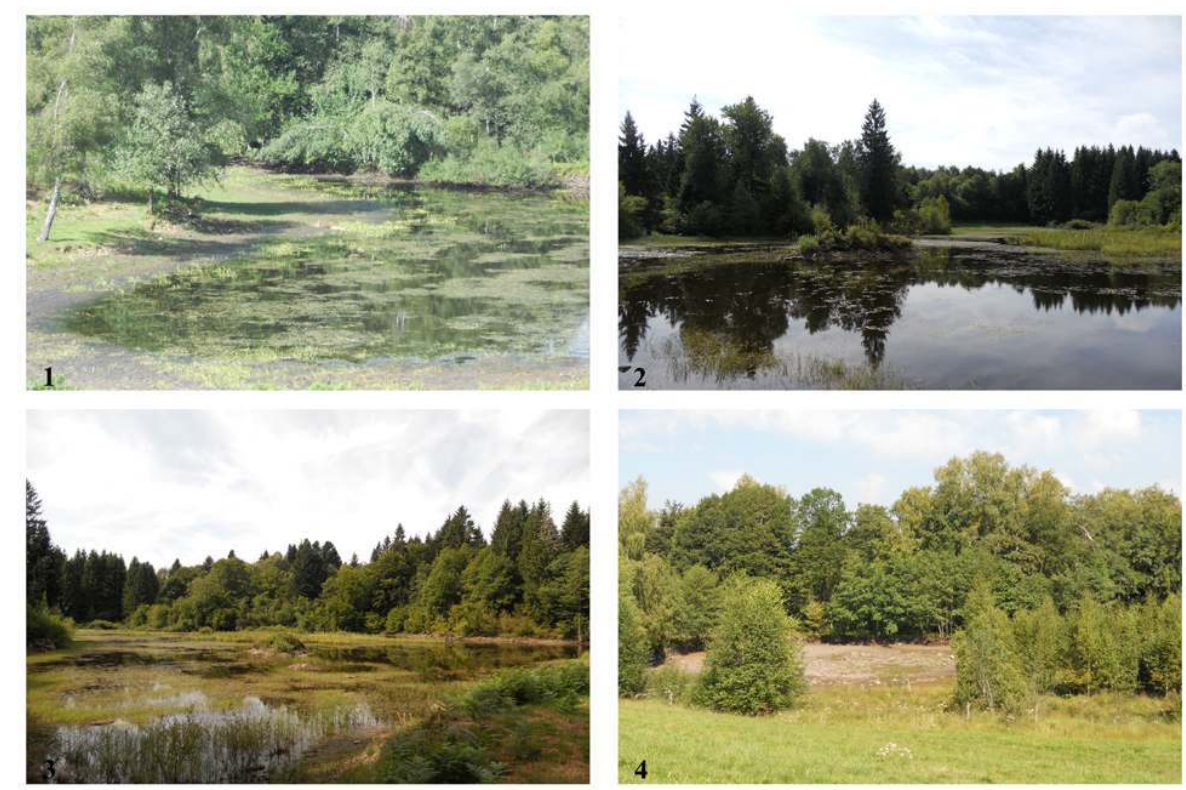

Le terroir des étangs du plateau se révèle particulièrement fragile. La faible profondeur en eau laisse «fleurir » la surface de l'étang et souligne l'origine de l'améngament d'anciennes tourbières et de zones marécageuses (photographies $n^{\circ} 1,2$ et 3 ). Ces vues illustrent les dynamiques en cours. Ainsi ces étangs, qui sont déjà repris dans un cycle naturel aboutissant à un nouveau stade de type tourbière. La photographie $\mathrm{n}^{\circ} 4$ montre l'abandon d'un étang et l'effacement de la zone humide.

Sources : A. et D. Mathis, 2014.

L'agro-sylvo-stagnosystème Mille Étang est aujourd'hui inscrit dans une mutation progressive. Les espaces agricoles ont désormais une «sole récréative ». Cette dernière, dans le cadre du plateau, s'est greffée sur un territoire en forte déprise mais à la forte image d'un "paysage nature ", dont le cœur systémique est constitué par les étangs. En effet, si leur rôle complémentaire à l'agro-sylvosystème est de plus en plus marginalisé, il faudrait alors considérer que la place paysagère des étangs est faible. C'est oublier, comme le suggère C. Juin Rialland (2009) que «Le paysage n'est plus simplement examiné comme une composante et une résultante des structures agraires, il exprime une vision esthétique pour ceux qui le fréquentent et qui entendent l'aménager à des fins ludiques ou contemplatives ». Comme pour les « Grandes Pêches » des étangs lorrains, le maintien de l'activité piscicole constitue aujourd'hui un facteur important de préservation des paysages d'étangs et, dans une certaine mesure, de leur patrimonialisation. 
Les étangs sont encore une masse d'eau, intégrée au système productif, objet ordinaire au sens de J-P. Deffontaines (2004) au cœur d'un terroir de moyenne montagne; ils sont devenus progressivement une marque de cette région, pour finalement constituer le facteur d'identité d'un « pays d'étangs ».

\section{B. Trouver une nouvelle médiance ${ }^{12}$ pour la « nature ordinaire »} traduit, pour ce territoire, l'émergence d'une identité renouvelée, reflet de son appropriation par une société urbaine, mais aussi la résilience d'un des aspects de la vie rurale. Cette dernière développe, temporairement, au bord de ces étangs un assolement touristique et de loisirs. Cette pratique repose en grande partie sur la perception du paysage. Toutefois les aménageurs, les habitants permanents, les décideurs ne perçoivent pas les mêmes rythmes de transformations et les cycles d'évolution du paysage aménagé " ouvert » à un retour à la nature suite à un processus de déprise. Finalement, le paysage est un vecteur intermédiaire, s'inscrivant dans un caractère immuable ou selon des cycles et des dynamiques. Quant aux aménageurs, ils se situent plutôt dans des démarches de production, de construction ou simplement d'entretien, voire d'abandon. Pour les différents observateurs, la perception descriptive de l'étang, qui traduit son « image » ou son « reflet » est différente et touche également au rapport sensible nature - culture. remarquables dans le cas du plateau par leur nombre ainsi que par leur inscription historique et fonctionnelle avec les autres activités du territoire. Leur valeur actuelle est liée au développement d'un assolement touristique et récréatif qui profite de l'importance de la déprise anthropique, de la bivalence nature/culture des étangs et de la forte image des espaces d'étangs comme étant des espaces authentiques, de calme, de pratiques agricoles traditionnelles, immuables, bien que parfois animés de profondes transformations. construite et raisonnée. Cette dernière est mise en œuvre par le PNRBV. À ce titre c'est une production collective qui relève d'une logique individuelle de protection et de préservation des terroirs d'étangs. Le paysage d'eau est un point d'appui à des projets de développement territorial qui induisent une sensibilisation à la valeur patrimoniale du paysage. C'est la raison pour laquelle la CC-1000 Étangs s'est engagée dans la définition d'un plan de paysage. Pour les habitants, il s'agit de définir collectivement un document contractuel autour d'un projet paysager pouvant servir d'axe pour l'avenir de leur territoire. Cette approche participative, liée à la médiation paysagère, comme ont pu le souligner S. Paradis et L. Lelli (2010), permet aux habitants de s'inscrire dans «la dialectique environnement/développement", et entrer ainsi dans une démarche de « développement territorial durable ».

L'entrée paysagère et descriptive qui porte plus particulièrement sur le faciès « étangs » Dans ce clivage conceptuel, les étangs, alors qu'ils sont un produit culturel et social, sont de plus en plus rangés parmi la "nature ordinaire », le "sauvage » qui constitue, ici, un facteur d'attractivité touristique. Isolés au cœur de forêts, les étangs peuvent être perçus comme un nouvel érème. Notamment pour des touristes ayant déjà une forte culture de la nature cela se traduit par des formes d'écotourisme où la valeur environnementale réelle ou sublimée est associée à des formes de découvertes patrimoniales, «sitologiques » et culturelles. C'est ce que traduisent les productions des routes et itinéraires touristiques, 
de découvertes et de randonnées qui traversent le plateau. La découverte des paysages, des dynamiques paysagères, des étangs, des tourbières, des cours d'eau est développée, largement commentée et expliquée.

Aujourd'hui, le cycle des étangs, perturbé par l'abandon des pêches et des assecs, porte à plus ou moins longue échéance à sa disparition. Les temporalités différentes entre le déclin des étangs et la dynamique végétale de la friche permettent d'afficher une résilience des plans d'eau. Ainsi sur ce plateau où une société agraire a produit des paysages d'eau originaux, la déprise agricole a entraîné, dans un premier temps, la survalorisation paysagère des étangs, peut-être sur la représentation empirique des eaux immobiles et stagnantes.

Les trouées paysagères des étangs constituent l'élément identitaire du plateau. Cette production permet d'inscrire ces derniers au cœur d'une démarche touristique et d'une pratique des loisirs et de la nature dans une forme de retour à la "nature " et au "sauvage ». L'enclavement de la région favorise la perception de dépaysement et d'isolement, de calme et de nature. Pour les étangs, malgré le sentiment immuable et intemporel que ces derniers véhiculent dans l'imaginaire, leur évolution naturelle est une menace à long terme sur ce modèle d'écotourisme ou d'éco-loisirs.

Le "désordre écologique » lié aux dynamiques des multiples faciès paysagers (forêts, étangs...) a favorisé pour l'instant un nouvel usage des étangs. Cette mutation fonctionnelle a implicitement donné de la valeur à ces lieux qui trouent le plateau forestier, au point d'en faire désormais un de ses marqueurs spécifiques. Toutefois, cette marque est-elle "durable»? À moyen terme, elle marque risque finalement de n'être plus qu'une trace réintégrée à une échelle de perception d'une nature "ordinaire", suivant les critères de perception que l'on peut y attacher. Comme souvent pour les pays d'étangs, la condition de la survie de ces derniers passe par la capacité des sociétés à les inscrire au sein d'une fonctionnalité qu'elle soit piscicole ou d'agrément. Cette fonctionnalité doit être suffisante économiquement pour rendre pérenne la structure sous peine de voir cette dernière remise en cause.

\section{Conclusion}

Le déclin économique et fonctionnel des vieux pays d'étangs modifie la perception de ces territoires. Désormais, c'est la valeur environnementale, écologique et sociale de ces derniers qui est mise en avant dans les projets territoriaux des Communautés de Communes et des Parcs Naturels Régionaux. Toutefois, le déclin fonctionnel entraîne irrémédiablement une évolution paysagère sensible. Pour le Plateau des Mille Étangs, cela correspond à la lente fermeture du paysage par la disparition des prairies et les reboisements, qu'ils soient organisés ou spontanés. Cette dynamique naturelle, conséquence de la déprise anthropique, annonce et provoque également, et à terme, la lente disparition des étangs non entretenus qui se comblent. Le phénomène d'atterrissement est lent par rapport aux stagnosystèmes de plaine en raison de la pauvreté des milieux et de la faiblesse de la production de matière organique. La dynamique de péjoration de la structure de l'étang, dans une lecture anthropique, favorise le retour au statut de tourbières dont la valeur écologique, en terme de biodiversité, est considérée comme nettement supérieure. Il n'appartient pas à cette étude de qualifier la valeur du milieu « agricole» et du milieu " naturel », soit du " rural » et du «sauvage » pour reprendre la réflexion menée par A. Berque (2011). L'érème du 
plateau, marqué par le déclin agricole et anthropique, illustre le glissement progressif du paysage et les enjeux concernant l'identité de ce dernier. L'effacement des étangs, que le processus s'inscrive dans le cycle de déprise agricole ou dans le cadre d'une politique destinée à rétablir les continuités écologiques, constitue un sujet de débat qui modifiera sensiblement le paysage à plus ou moins longue échéance.

Sur ce plateau où la place du "sauvage » est désormais importante, le maintien de « corridors anthropiques » se manifeste par le projet touristique et de loisirs autour des pôles des étangs. Ce paysage est le produit d'une médiation entre les hommes et la nature. Il constitue l'héritage d'une société qui a rationalisé son territoire, ses ressources pour en tirer de quoi assurer sa subsistance. Laisser le cycle dynamique de restauration des tourbières par effacement spontané ou décidé souligne une démarche volontariste qui traduit un choix entre deux milieux connexes. Aujourd'hui les paysages du «plateau » sont repris au sein de projets paysagers et de démarches de valorisations par les "aménageurs d'espace", qu'il s'agisse du PNRBV ou de la CC-1000 Étangs. Parmi ces démarches, il reste un débat qui concerne la place à accorder à la gestion de la ressource en eau d'un territoire. Le savoir-faire mis en œuvre doit-il être conservé et par là-même faut-il réaffirmer les identités des territoires d'eau ? Ainsi, la valeur écologique des espaces d'eau au sein de l'hydrosystème devrait tenir compte également des formes de complémentarités établies par les anciens socio-systèmes car ces derniers ont mobilisé et construit une gestion cohérente et solidaire de la ressource en eau valorisant un territoire et ses périphéries.

\section{BIBLIOGRAPHY}

Ardillier-Carras F., 2009, « La maîtrise de l'eau pour l'agriculture en Limousin. Un exemple de gestion de la ressource en moyenne montagne océanique », Norois [En ligne], 210 | 2009/1, URL : http://norois.revues.org/2746

Benarrous R., Marinval M-C., 2006, « La Carpe (Cyprinus carpio), cette orientale qui séduit l'Occident au Moyen Age », Derex J.-M., (textes réunis par), La production des étangs du Moyen Age à l'époque contemporaine,Actes de la journée d'étude 2005 du Groupe d'Histoire des Zones Humides, 120 p., p.9-22.

Berque A., 2000, Médiance, de milieux en paysages, Paris, Belin GIP Reclus, 161 p.

Berque A., 2011, « le rural, le sauvage, l'urbain », Études Rurales, n¹87, janvier-juin 2011, p.51-62.

Bonnemaison J., 1992, « Le territoire enchanté. Croyances et territorialités en Mélanésie », Géographie et culture, $\mathrm{n}^{\circ} 3, \mathrm{p} .72-88$.

Brossier J., et al.,2008 - Quels paysages avec quels paysans ? Les Vosges du Sud à 30 ans d'intervalle, éditions Quæ, $126 \mathrm{p}$.

Cabouret M., 1999, L'irrigation des prés de fauche en Europe occidentale, centrale et septentrionale. Essai de géographie historique. Préface d'Erik Bylund, Editions Karthala, Paris, $1999,319 \mathrm{p}$. 
Chiffre E. et Mathis D., 2015, « Prise en compte des hydrosystèmes hérités par les parcs naturels régionaux de Lorraine et du Morvan ", Territoire en mouvement Revue de géographie et aménagement [En ligne], 25-26, 2015, mis en ligne le 31 mars 2015. URL : http://tem.revues.org/2764

Clément G., 2004, Manifeste pour le Tiers paysage, Paris, Éditions Sujet/Objet, 70 p.

Deffontaines J-P., 2004, « L'objet dans l'espace agricole. Le regard d'un géoagronome ». Nat.Sci. Soc ., 12, p.299-304.

Delignières V., 1998, « L'authenticité des territoires ruraux touristiques en question Réflexions au sujet de l'Auxois et du Morvan ", Revue Géographique de l'Est, tome XXXVIII, n 3, p.103-116.

Dupré L., 2005, « Des friches : le désordre social de la nature », Terrain [En ligne], 44 | mars 2005, mis en ligne le 20 avril 2005, URL : http://terrain.revues.org/2488.

Flageollet J-Cl., 2002, Sur les traces des glaciers vosgiens, Paris, CNRS éditions, $212 \mathrm{p}$.

Ghiotti S., 2007, Les territoires de l'eau. Gestion et développement en France, Paris, CNRS Editions, 246 p.

Guillaud D., 2008, «L'archéogéographie : pour une reconnaissance du passé dans l'espace », EchoGéo [En ligne], 4 | 2008, mis en ligne le 01 mars 2008. URL : http://echogeo.revues.org/2278 Husson J-P., Envies de campagne. Les territoires ruraux français, Paris, Ellipses, 2007 p. Juin-Rialland C., 2009, « De la géographie rurale à l'analyse des paysages : le témoignage d'un chercheur ", L'Information géographique, 2009/1 Vol. 73, p.17-29.

Le Caro Y., 2012, «Les agriculteurs et le partage de l'espace agricole pour des usages récréatifs », in Papy F., Mathieu N., et Férault Ch. (dir.), Nouveaux rapports à la nature dans les campagnes, Editions QUÆ, 19 p., p.101-117.

Luginbühl Y., 2012, La mise en scène du monde, CNRS Editions, 432 p.

Mathis D., 2014, « Recomposition territoriale d'un espace rural flou : l'exemple du « Pays des Étangs » en Moselle », Territoire en mouvement Revue de géographie et aménagement [En ligne], 22 | 2014, mis en ligne le 15 juin 2014. URL : http://tem.revues.org/2415

Mathis D., 2015, « Dynamiques des paysages du massif du Donon et de ses périphéries mosellanes ", in Corvol A. et al., (dir.), Forêt et montagne, GHFF, Paris, Editions L'Harmattan, p.383-398.

Paradis S. et Lelli L., 2010, « La médiation paysagère, levier d'un développement territorial durable? ", Développement durable et territoires [En ligne], Vol. 1, nº 2 | Septembre 2010, mis en ligne le 17 septembre 2010. URL : http://developpementdurable.revues.org/8548

Perrier A., 1925, «L'évolution industrielle des vallées vosgiennes de la Haute-Saône », Annales de Géographie, t. 34, n¹89. p.272-277.

\section{NOTES}

1. Ne pas confondre avec la réserve naturelle nationale de Chérine située au sein du Parc régional de Brenne (Indre) qui a été rebaptisée également « Pays des Mille Étangs ».

2. Il faut interpréter ce nombre de mille comme une valeur indicative.

3. Lévêque Ch., Muxart T., «Anthoposystème", Hypergéo, consulté le 18/03/2014, www.hypergéo.eu/spip.php?article270\# 
4. Communauté de communes des 1000 Etangs, consulté le 05 décembre 2015, http:// www.cc-1000etangs.fr/tourisme_et_loisirs/paysages.htm

5. Adapté des échelles des hydrosystèmes et des limnosystèmes, le terme de stagnosystème permet une qualification plus pertinente des systèmes des étangs qui structurent des bassins versants formant soit de vastes plans d'eau de plusieurs centaines d'hectares comme pour le Pays des Étangs en Lorraine (Chiffre et Mathis, 2015), soit des territoires marqués par l'omniprésence des étangs comme pour les Mille Étangs.

6. Comme dans d'autres territoires d'étangs, on assiste ici à l'apparition de formes de résidences secondaires composées de caravanes ou de mobil home parfois indurés par l'adjonction d'abris divers. Toutefois, la petite taille des étangs favorise ici l'individualisme de ce mitage du foncier.

7. La moitié des étangs a moins de 50 ares.

8. Le moulin Bégeot à Mélisey, les vestiges du moulin de La Mer... illustrent encore l'important développement des activités pré-industrielles.

9. La scierie Martin à Servance (le Haut-du-Them) constitue aujourd'hui un témoin de cette activité. Ce haut-fer réhabilité avec le soutien du PNRBV, qui anime le site, s'intègre à un projet d'écotourisme des Vosges Saônoises. Le PNRBV a placé différents sites témoins en réseau autour de la thématique «force hydraulique ».

10. Bien qu'ayant cessé son activité, la forge a conservé ses machines comme témoins du patrimoine technique et industriel. Elle est entrée dans un processus de patrimonialisation et de mise en valeur comme en témoigne son inscription à l'inventaire.http://www.culture.gouv.fr/ documentation/memoire/VISITES/corravillers/index.html

11. La chaine des étangs constitue aujourd'hui un corridor écologique, plus favorable, malgré les retenues et les « seuils », que les tourbières et marais installés dans les « creux » qui forment des stations isolées.

12. En référence à l'ouvrage d'A. Berque (2000).

\section{ABSTRACTS}

The "Plateau des Mille Etangs" is an old agro-sylvo-fishing country of mountain. Nowadays, this territory of water, shaped by glaciers and developed by man, knows a co-evolution between natural dynamics caused by the anthropogenic abandonment and territorial projects of the Parc Naturel Regional des Ballons des Vosges and EPCI du plateau des Mille Etangs based on the valorisation of an anthropico-natural heritage. Natural dynamics, nature protection, conservation of the anthropogenic legacies are important issues for this territory and influence the evolution of these landscapes of ponds.

Le Plateau des Mille Étangs est un vieux pays agro-sylvo-piscicole de moyenne montagne. Aujourd'hui, ce territoire d'eau, façonné par les glaciers et aménagé par l'homme, connaît une co-évolution entre une dynamique naturelle provoquée par la déprise anthropique et les projets du Parc Naturel Régional des Ballons des Vosges et des EPCI du plateau des Mille Étangs fondés sur la valorisation d'un patrimoine naturel et humain. Dynamique naturelle, protection de la nature, conservation des héritages anthropiques constituent des enjeux importants pour ce territoire et conditionnent l'évolution de ces paysages d'étangs. 
Das "Mittelgebirge Plateau des Mille Etangs" ist eine alte Weiherlandschaft, die sich im Zuge der Vergletscherungen ausgeformt hat und später vom Menschen eingedeicht wurde. Heute kennt dieses Wassergebiet, eine durch menschliche Abkehr begünstigte dynamische natürliche Entwicklung. Der Ausbau des regionalen Naturparks « les Ballons des Vosges » und die Projekte im Gebiet « des Mille Etangs », die zeitgleich erfolgen, sollen die Wahrung des Naturerbes und die Förderung einer Teichlandschaft ermöglichen.

INDEX

Keywords: Vosges Saonoises, regional park, fish farming landscape, tourism Schlüsselwörter. Vosges Saonoises, Regionalpark, Teichlandschaft, Tourismus Mots-clés: Vosges Saônoises, parc régional, paysage de pisciculture, tourisme

\section{AUTHOR}

\section{DENIS MATHIS}

Docteur en géographie, chercheur associé - Université de Lorraine.Laboratoire d'Observation des TERRitoires (LOTERR)23, boulevard Albert $1^{\mathrm{er}}$ - BP 33-97 - 54015 NANCY CEDEX 